\title{
The pathophysiology of increased hepatic IGF-1 expression in an ovine model of polycystic ovary syndrome (PCOS)
}

\author{
Siemienowicz K. ${ }^{1}$, Boswell L. ${ }^{1}$, Carr D.J. ${ }^{2}$, Mina T.H. ${ }^{1}$, Connolly F. ${ }^{1}$, Rae M.T. ${ }^{3}$, Duncan W.C. ${ }^{1}$
}

${ }^{1}$ MRC Centre for Reproductive Health, The University of Edinburgh, Edinburgh, UK, 2 University College London, London,

UK and ${ }^{3}$ Edinburgh Napier University, Edinburgh, UK.

\section{Introduction}

An adverse fetal environment can result in epigenetic programming events that predispose to adult disease ${ }^{1}$. Animal studies showed that prenatal androgenisation programmes a polycystic ovary syndrome (PCOS)-like phenotype in adult offspring, including metabolic, reproductive and endocrine abnormalities ${ }^{2}$. Recently we reported that exposure of pregnant sheep to increased concentrations of testosterone during midgestation results in metabolic changes in adult offspring, including increased insulin secretion to glucose load and increased hepatic IGF ${ }^{3}$. Here, we focus on investigating the molecular pathophysiology of the IGF1 increase.

\section{Aims}

- To determine if altered IGF1 is a consequence of prenatally programmed differential gene methylation.

- To determine if IGF1 increase is a consequence of altered growth hormone $(\mathrm{GH})$ secretion or action.

- To determine if contemporaneous androgen exposure is involved in the IGF1 increase.

\section{Methods}

Pregnant Scottish Greyface ewes were treated biweekly with either 100mgs of testosterone propionate (TP) or vehicle control $(C)$ from $d 62-102$ of gestation $(C=5$, $\mathrm{TP}=9$ ). Females offspring were assessed at 11 months of age.

In a separate experiment 3 years old normal female Scottish Greyface ewes were treated biweekly with either 100mgs of testosterone propionate (TP) or vehicle control (C) for two weeks $(C=5, T P=5)$.

Livers and pituitaries of experimental animals were snap frozen for qRT-PCR analysis and also fixed in bouins for subsequent paraffin wax embedding and immunohistochemistry. Serum GH was measured by ELISA. Gene methylation was measured by pyrosequencing.

\section{Results}

\section{IGF-1 methylation}

Increased hepatic IGF1 is not a consequence of prenatally programmed hypomethylation as assessed by IGF1 CpG methylation using pyrosequencing.

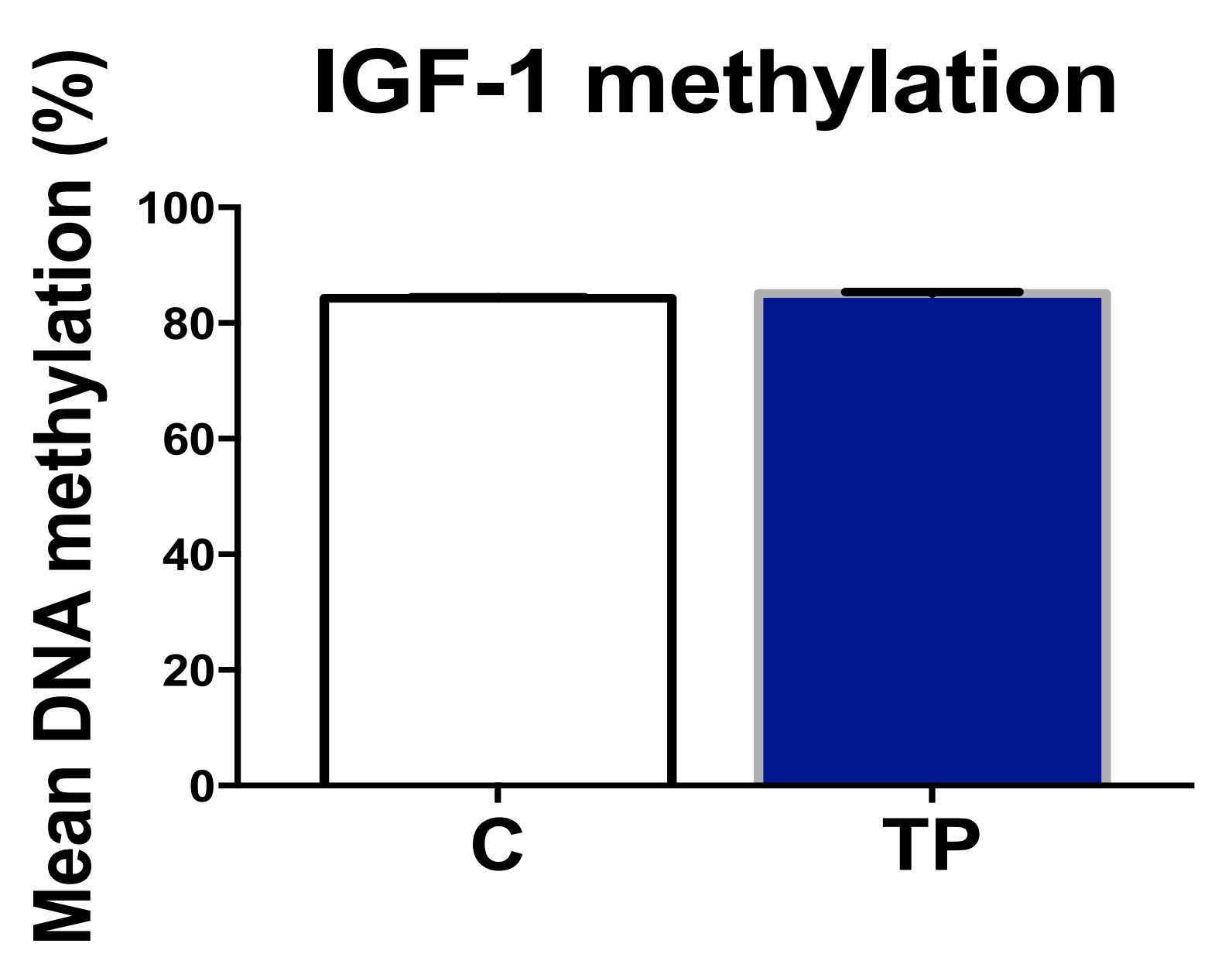

\section{Growth hormone}

The prenatally programmed primary hyperinsulinaemia and subsequently augmented IGF1 is not a consequence of altered growth hormone $(\mathrm{GH})$ secretion or action. There was no difference in pituitary GH mRNA expression, number of somatotrophs assessed by immunohistochemistry or serum GH concentrations.
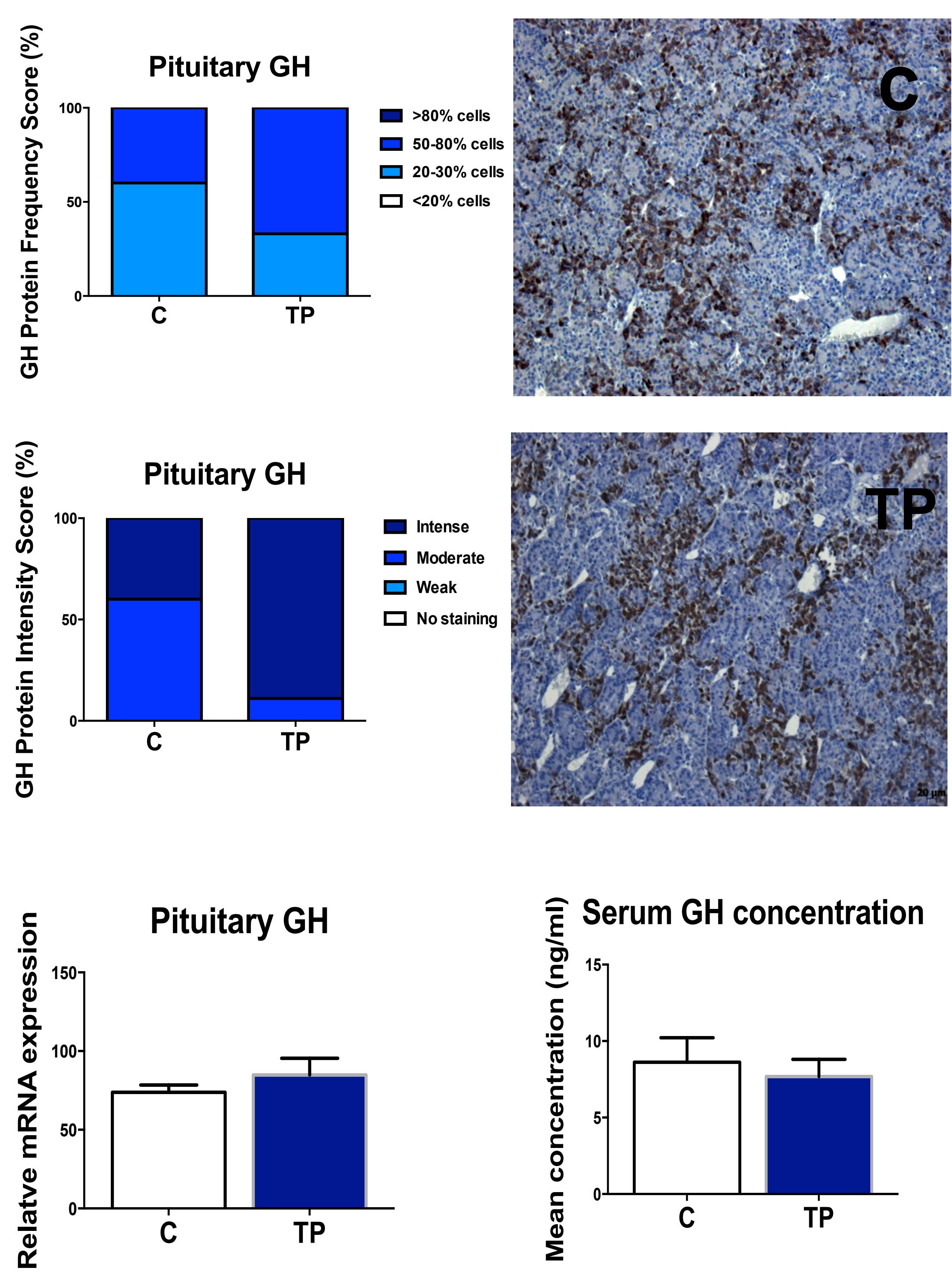

In addition there were no differences in hepatic GHR or expression of other $\mathrm{GH}$ regulated genes including HNF6/ONECUT1, G6PC and SLC2A2.
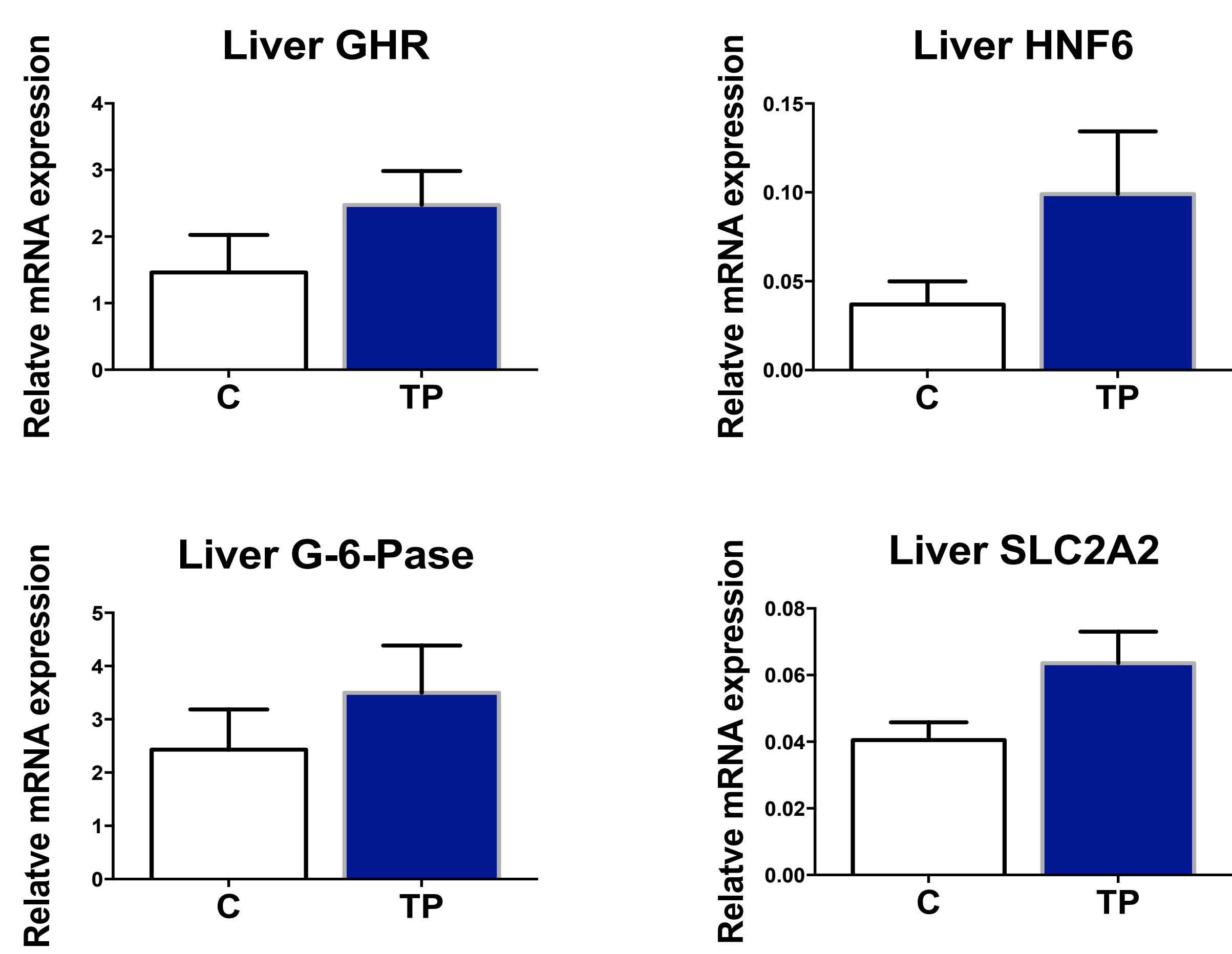

\section{Effect of contemporaneous androgen exposure}

Prenatally androgenised sheep express higher hepatic $A R^{3}$ and have increased capacity for ovarian and adrenal androgen synthesis ${ }^{4}$, therefore to determine if observed increase of hepatic IGF1 was a result of prenatal androgenisation or contemporaneous androgen exposure a separate cohort of adult sheep was utilised.

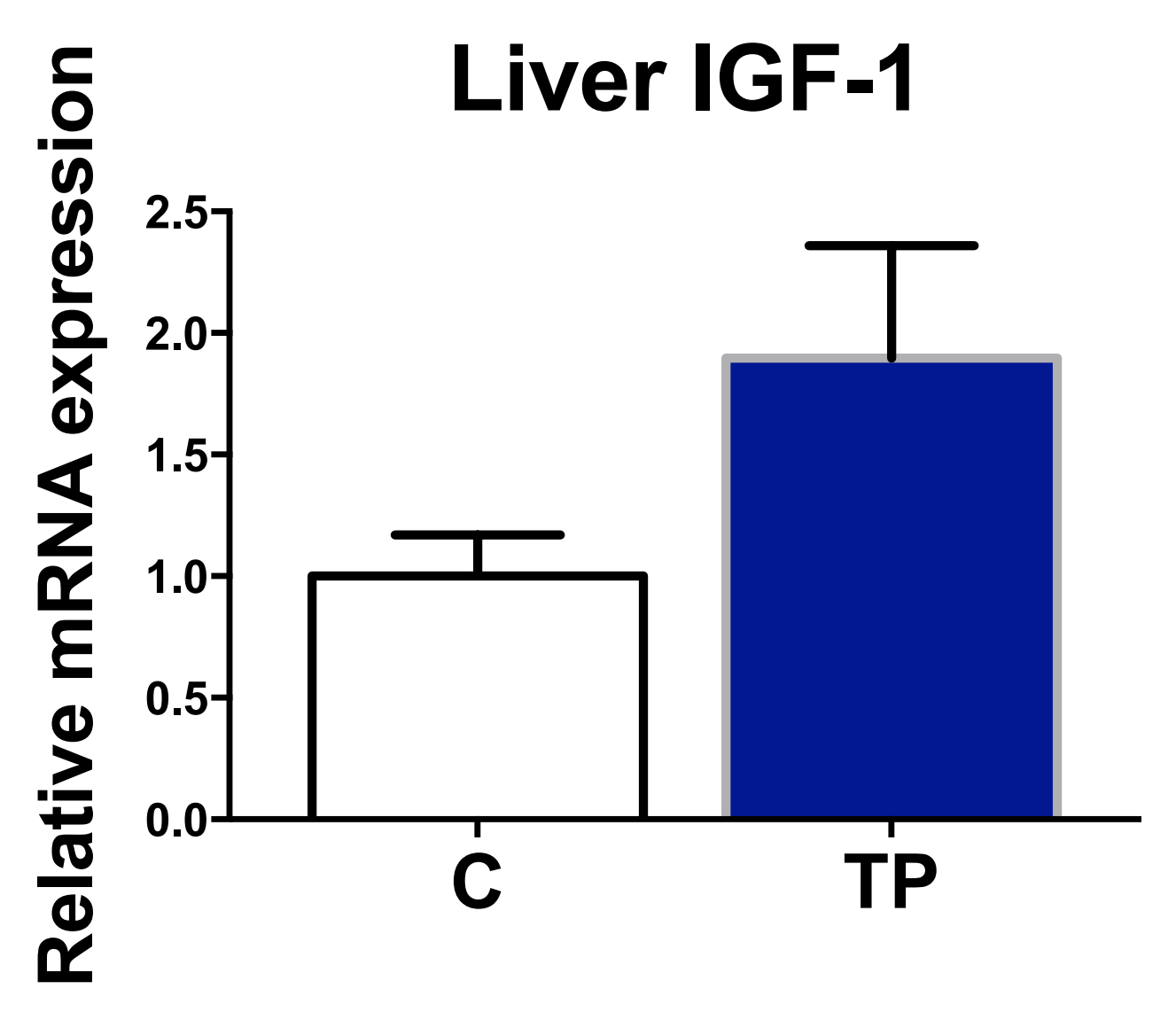

The regimen of two week testosterone treatment did not significantly alter hepatic IGF1 expression.

\section{Conclusion}

- Prenatal androgenisation of female fetuses but not a contemporaneous testosterone exposure of adult female sheep increases hepatic IGF1 in adult ovine females.

- The increase is not a consequence of altered growth hormone $(\mathrm{GH})$ secretion or action nor hypomethylation of hepatic IGF1 gene.

- To date the pathophysiology of increased hepatic IGF1 in the ovine model of PCOS is unclear.

\section{References}

1. Jirtle R and Skinner M (2007) Environmental epigenomics and disease susceptibility. Nature Reviews Genetics 8: 253-262.

2. Franks S (2012) Animal Models and the Developmental Origins of Polycystic Ovary Syndrome: Increasing Evidence for the Role of Androgens in Programming Reproductive and Metabolic Dysfunction. Endocrinology. 153: 2536-2538.

Hogg K, Wood C, McNeilly A and Duncan C (2011) The In Utero Programming of Increased Maternal Androgens and a Direct Fetal Intervention on Liver and Metabolic Function in Adult Sheep. Ploss One. 6: e24877

4. Hogg K, Young J, Oliver E, Souza C, McNeilly A and Duncan C (2012) Enhanced thecal androgen production is prenatally programmed in an ovine model of polycystic ovary syndrome. Endocrinology. 1:450-461. 